\title{
Trade-off between salt secretion and gas exchange by stomata in the leaves of Glycyrrhiza uralensis
}

\author{
Peng You Chen, Miao Ma* and Ling yu Shi \\ Ministry of Education Key Laboratory of Xinjiang Phytomedicine Resource Utilization, College of Life Sciences, Shihezi University, \\ Xinjiang 832003, The People's Republic of China
}

\begin{abstract}
Our previous study found that stomata in the leaves of Glycyrrhiza uralensis (licorice) could secrete salt crystals. In theory, secretion of salt should affect the normal functioning of stomata, thereby affecting the growth and development of $G$. uralensis; however, its population grows well. We suspect that there may be a trade-off between stomatal salt secretion and gas exchange from the leaves at different positions. Therefore, we compared stomatal salt secretion capacity, chlorophyll content, anatomical structure, net photosynthetic rate and stomatal conductance from the leaves at different positions of licorice. The stomata of lower leaves exhibited strongest salt secretion capacity, whereas the stomata of upper leaves did not secrete any salt. Additionally, the upper and middle leaves had significantly higher chlorophyll content than the lower leaves. The arrangement of mesophyll cells in the upper leaves was densest, and that in the lower leaves was least dense. The net photosynthetic rate and stomatal conductance in the upper leaves were highest, and those in the lower leaves were lowest. We conclude that the stomata of upper leaves are mainly used for gas exchange. In contrast, stomata of lower leaves, showing weak photosynthesis, are responsible for secreting excessive salt to maintain the inner ion balance and ensure normal metabolism in $G$. uralensis.
\end{abstract}

Keywords: Licorice, stomata, salt secretion.

RooTs and rhizomes of licorice (Glycyrrhiza uralensis Fisch) are Chinese traditional herbs with various pharmacological effects ${ }^{1}$, such as alleviating bacteriostasis ${ }^{2}$, cough $^{3}$ and spasmolysis ${ }^{4}$; combatting viruses ${ }^{5}$; alleviating inflammation and removing toxins ${ }^{6}$; blocking radiation effects ${ }^{7}$ and cancer $^{8}$, thus making these herbs popular in the pharmaceutical world. Because G. uralensis generally grows in calcareous grasslands ${ }^{9}$ and salt-containing lowland meadows, it has been traditionally considered a halophyte. Salinity is an essential environmental factor that affects plant growth and development, and salt stress can directly affect plant growth and development,

*For correspondence. (e-mail: mamiaogg@126.com) reduce photosynthetic rates, and inhibit photosynthesis, thereby altering the pigment content of plant leaves and indirectly affecting its growth ${ }^{10}$. Till date, though some experimental studies on the salt tolerance of G. uralensis seeds have been performed ${ }^{11}$, the mechanism of adaptation of licorice to salted soil has not yet been reported.

Stomata are the channels through which plants exchange gases with the outside world. The outside $\mathrm{CO}_{2}$ enters the plant leaves through stomata, which are also essential for internal water vapour and $\mathrm{O}_{2}$ elimination. The process of leaf photosynthesis is automatically regulated by stomata, which plays an important role in respiration, water metabolism ${ }^{12}$, immune system $^{13}$, and the maintenance of leaf-surface temperature of plants ${ }^{14}$. Therefore, maintaining the normal function of stomata is vital to the healthy growth and development of plants.

Salt secretion is a critical salt tolerance mechanism in plants. Studies have shown that salts in plants are generally secreted by salt glands, glandular trichomes, vesicles, and other salt-secreting structures ${ }^{15}$. Previous studies have found that salt glands and stomata on the leaves of G. uralensis living in high-salt habitats have strong salt secretion capacity, resulting in secretion of large amounts of salts to the leaf surface. Secretion of salt crystals can block stomata, thereby affecting the normal regulation of stomatal opening, gas exchange between plant and its external environment, and $\mathrm{CO}_{2}$ assimilation and water transpiration of leaves. These effects can increase leaf temperature and decrease photosynthesis-related enzyme activity $^{16}$, which may alter the growth and development of $G$. uralensis and accumulation of secondary metabolites. However, G. uralensis populations grow well and do not show any symptoms of injury. Therefore, we hypothesized that there may be a trade-off or balance between gas exchange and salt secretion in the stomata of licorice leaves.

Accordingly, in this study, we aimed to determine this functional trade-off mechanism of licorice leaves, stomata by comparing their abilities of salt secretion and photosynthetic gas exchange at their different positions on the plant body, to provide evidence for the adaptation mechanisms of plant in salt-containing soil. 


\section{Materials and methods}

\section{Sample area and materials}

G. uralensis was sampled from the salinized lowland meadow $\left(47^{\circ} 51^{\prime} \mathrm{N}, 86^{\circ} 51^{\prime} \mathrm{E}\right)$ in Altay, Xinjiang, China, which exhibits a temperate continental climate with a long, cold winter and short, mild summer. Although G. uralensis population in the sample area lives under extremely saline soil conditions, they grow normally and fulfill their lifecycle successfully. The field experiments were started in July 2016.

\section{Electrical conductivity and $p H$ of the soil}

A five-point method was used for stratified random sampling of soil, and each point was divided into three layers: 0-10, 10-20 and 20-30 cm. Soil samples from the same layer were sealed into a bag after being mixed and then brought back quickly to the laboratory for airdrying. The samples were then ground and sieved through $1 \mathrm{~mm}$ mesh for determination of soil conductivity. Soil conductivity and $\mathrm{pH}$ was determined using a DDSJ-308A (Shanghai Tuxin Electronic Technology Co, Ltd) conductivity meter $(k=0.964)$ in a $1: 5$ soil/water suspension. The methods for soil agrochemical analysis described by $\mathrm{Bao}^{17}$ were used to evaluate the grade of soil salinization, and the same soil sample was evaluated three times. The results were presented as averages.

\section{Stomatal salt secretion from the leaves at different positions}

Six individual plants of G. uralensis in an open area were randomly selected and divided into three parts equally, i.e. upper, middle and lower, according to height (plant height, $75 \mathrm{~cm}$ ). Then, one healthy and mature leaf from each of these three sections were selected randomly, cut into pieces $(0.5 \mathrm{~cm} \times 0.5 \mathrm{~cm})$, and placed in FAA solution $(70 \%$ alcohol : glacial acetic acid : formaldehyde $=18: 1: 1)$ for $48 \mathrm{~h}$. Subsequently, the leaf pieces were fixed on the sample table and subjected to ion sputtering gold plating. Samples were then observed with a scanning electron microscope (SU8010, Hitachi High-tech Company) and photographed. Chemical composition of secretions from the stomata was detected by energy dispersive spectrometer (7353, Oxford) to determine whether they are salts. Five fields $(600 \times$ magnification) were selected randomly for enumeration of the total number of stomata and the number of salt-secreting stomata, and the percentages of salt-secreting stomata were calculated to determine the average.

\section{Chlorophyll content of the leaves}

The upper, middle and lower parts of the same fullydeveloped, healthy leaves used for microscopic analysis were selected randomly. The main veins were then removed, and the remnants were cut into small pieces and mixed well. Next, $0.25 \mathrm{~g}$ of the chopped leaves was accurately weighed and ground into homogenate with $95 \%$ ethanol $(10 \mathrm{ml})$ in the dark until the tissue colour turned white. The final chlorophyll concentrations were measured according to Callejas et al. ${ }^{18}$.

\section{Anatomical structure of the leaves}

Healthy and fully expanded leaves growing at different positions were selected randomly, cut into small pieces $(0.5 \mathrm{~cm} \times 0.5 \mathrm{~cm})$, and placed in FAA solution for $48 \mathrm{~h}$. Transverse sections of the leaves $(8 \mu \mathrm{m}$ thick) were prepared using conventional paraffin sectioning. The sections were stained with safranin and fast green, sealed with optical resin, observed with an Olympus BX51 light microscope, and photographed with an Olympus DP70 system.

\section{Photosynthetic capacity of the leaves}

In an open field, six individual $G$. uralensis plants with similar heights and crown widths were selected, and one healthy and completely expanded leaf was selected. The net photosynthetic rate $\left(P_{n}\right)$ and stomatal conductance $\left(G_{s}\right)$ of the leaves were determined using a LI-6400 portable photosynthesis analyzer (Beijing Ecotek Technology Co, Ltd) at $2 \mathrm{~h}$ intervals. The measurements were done between $08: 00$ and 20:00 on a sunny, cloudless day and each leaf was evaluated three times to obtain the average value.

\section{Data analysis}

The data were analysed using SPSS 19.0. The conductivity among different soil layers and differences in chlorophyll, net photosynthetic rate and stomatal conductance among the upper, middle and lower leaves were analysed by one-way analysis of variance. Duncan's multiple range tests were used to detect the significance levels of differences among treatments $(P<0.05)$.

\section{Results}

\section{Electrical conductivity and $p H$ value of the soil}

The results showed that the conductivity of different soil layers decreased significantly as the soil depth increased. According to the standard in-soil agrochemical analysis $\operatorname{method}^{17}$, the $0-10 \mathrm{~cm}$ soil layer exhibited heavy salinization, and the $10-20 \mathrm{~cm}$ and $20-30 \mathrm{~cm}$ layers could also be classified as salinized soil. Since the $\mathrm{pH}$ value of each soil layer was above 10 , the soil is alkaline (Table 1). 

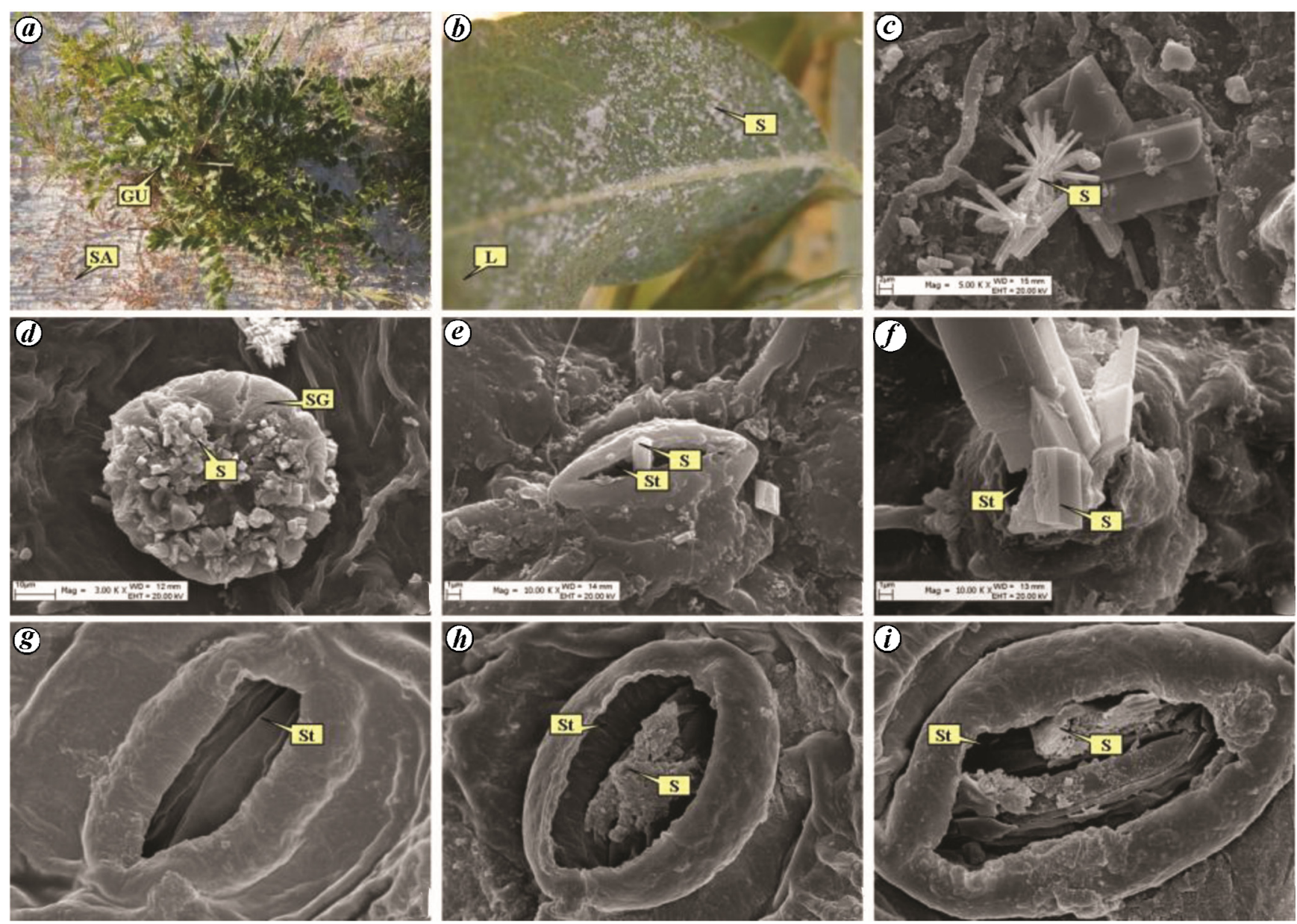

Figure 1. Glycyrrhiza uralensis in saline soil and its salt-secreting gland and stomata. GU, G. uralensis; L, leaf; S, salt; SA, saline-alkaline soil; $\mathrm{SG}$, salt gland; St, stomata. $\boldsymbol{a}$, Licorice on saline-alkaline soil; $\boldsymbol{b}$, Salt on leaf surface of licorice; $\boldsymbol{c}$, Salt crystals on the leaf surface (5000×); $\boldsymbol{d}$, Salt-secreting glands $(3000 \times) ; \boldsymbol{e}, \boldsymbol{f}$, Salt-secreting stomata $(10000 \times) ; \boldsymbol{g}$, Stomata on the upper leaf $(15000 \times) ; \boldsymbol{h}$, Stomata on the middle leaf $(15000 \times) ; \boldsymbol{i}$, Stomata on the lower leaf $(15000 \times)$.

Table 1. Conductivity and $\mathrm{pH}$ value of the soil

\begin{tabular}{lcc}
\hline Soil layer/cm & Mean value $\mathrm{EC}_{25} /(\mathrm{dS} / \mathrm{m})$ & $\mathrm{pH}$ value \\
\hline $0-10$ & $8.49 \pm 0.03 \mathrm{a}$ & $10.33 \pm 0.03 \mathrm{a}$ \\
$10-20$ & $3.51 \pm 0.02 \mathrm{~b}$ & $10.29 \pm 0.03 \mathrm{a}$ \\
$20-30$ & $3.42 \pm 0.01 \mathrm{c}$ & $10.20 \pm 0.02 \mathrm{~b}$ \\
\hline
\end{tabular}

\section{Comparison of stomatal salt secretion among the leaves at different positions}

Field observations revealed that salts were secreted by the salt glands and stomata onto the leaf surface (Figure $1 a-f)$, with significant differences in salt secretion among the upper, middle and lower leaves (Figure $1 g-i$; Table 2). Among the leaf regions, the upper leaf stomata were permeable, and no salt accumulation was observed (Figure $1 \mathrm{~g}$ ). In contrast, the stomata of lower leaves had strong salt secretion capacity, resulting in accumulation of large amounts of salts, with some stomata even being completely blocked by them (Figure $1 i$ ). Additionally,
$78 \%$ of the stomata in the middle leaves could secrete salt; however, the secretion was weak, with only a small amount of salt accumulating in these leaves (Figure $1 h$ ). These results indicated that the salt secretion in G. uralensis was mainly achieved by the lower leaves. The results of the energy spectrum analysis showed that the secretions discharged from the stomata contained various components (Figure 2), with the metal elements mainly being $\mathrm{K}$ and $\mathrm{Ca}$, and the non-metal elements, $\mathrm{S}$ and $\mathrm{O}$.

\section{Comparison of chlorophyll content among the leaves at different positions}

Chlorophyll content is closely related to plant photosynthesis, with higher chlorophyll content reflecting stronger leaf photosynthetic capacity. Our study showed that the chlorophyll content in the upper and middle leaves of $G$. uralensis was significantly higher than that in the lower leaves, and that there were no significant differences in the content between upper and middle leaves (Table 3). Thus, the upper and middle leaves of G. uralensis in the 
Table 2. Comparison of the percentage of salt-secreting stomata among leaves at different positions

\begin{tabular}{lcl}
\hline $\begin{array}{l}\text { Location of leaf } \\
\text { on the stem }\end{array}$ & $\begin{array}{c}\text { Percentage of } \\
\text { salt-secreting stomata (\%) }\end{array}$ & $\begin{array}{c}\text { Salt-secreting } \\
\text { degree of stomata }\end{array}$ \\
\hline $\begin{array}{l}\text { Upper leaf } \\
\text { Middle leaf }\end{array}$ & 0 & Without secreted salt \\
Lower leaf & 78 & With a small amount of secreted salt \\
\hline
\end{tabular}

Table 3. Comparison of chlorophyll content among leaves at different positions

\begin{tabular}{lccc}
\hline Leaf position & $\begin{array}{c}\text { Chlorophyll } a \\
(\mathrm{mg} / \mathrm{g})\end{array}$ & $\begin{array}{c}\text { Chlorophyll } b \\
(\mathrm{mg} / \mathrm{g})\end{array}$ & $\begin{array}{c}\text { Chlorophyll } a+b \\
(\mathrm{mg} / \mathrm{g})\end{array}$ \\
\hline Upper leaf & $9.13 \mathrm{a}$ & $2.609 \mathrm{a}$ & $11.739 \mathrm{a}$ \\
Middle leaf & $9.222 \mathrm{a}$ & $2.756 \mathrm{a}$ & $11.978 \mathrm{a}$ \\
Lower leaf & $7.45 \mathrm{~b}$ & $1.339 \mathrm{~b}$ & $8.789 \mathrm{~b}$ \\
\hline
\end{tabular}

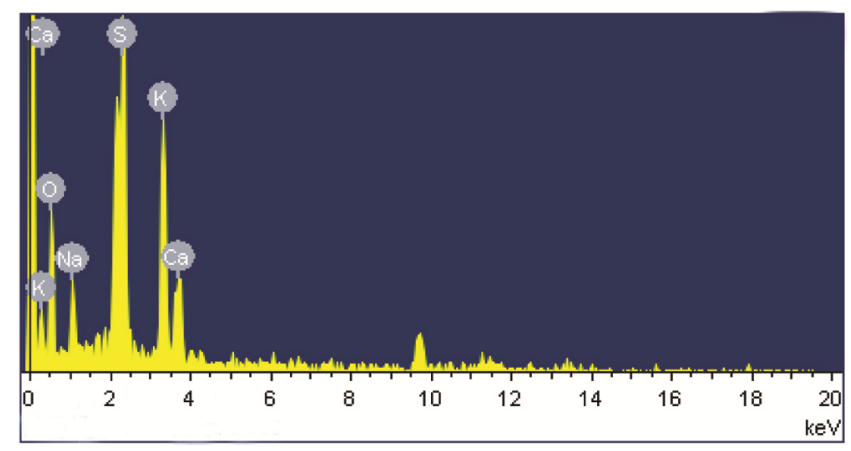

Figure 2. Energy spectrum analysis of secretions from stomata of $G$. uralensis.

salinized lowland meadow may have stronger photosynthetic capacity than the lower leaves because of their higher chlorophyll content.

\section{Comparison of anatomical structures of the leaves at different positions}

The thicknesses of the leaves at different positions were similar (Figure 3); however, the differentiation and density of the mesophyll cells differed. The arrangement of mesophyll cells in the upper leaves was the densest; in the middle leaves, moderate; whereas that in the lower leaves was sparse.

\section{Comparison of photosynthesis among the leaves at different positions}

The patterns of diurnal variation in the net photosynthetic rate $\left(P_{n}\right)$ of leaves at different positions were similar, exhibiting a typical bimodal curve and an apparent 'photosynthesis lunch break' phenomenon. However, there were significant differences in the photosynthetic capacity among the leaves at different positions (Figure 4). Notably, $P_{n}$ of the upper leaves was always the largest, followed by that of the middle leaves, while that of the lower leaves was the smallest. The diurnal variation pattern of stomatal conductance $\left(G_{s}\right)$ in the upper leaves also showed a bimodal curve; the conductance gradually increased from $08: 00$ onwards, with two peaks at 10:00 and 16:00, consistent with the diurnal variation pattern of $P_{n}$. In contrast, the diurnal variation patterns of $G_{s}$ in the middle and lower leaves were typical unimodal curves. Similarly, $G_{s}$ of the upper leaves was always the highest, followed by that of the middle leaves, and $G_{s}$ of the lower leaves was always the lowest. The $G_{s}$ values were significantly different among the leaves at different positions.

\section{Discussion}

Leaves are the main photosynthetic organs of the plants and the main exchange point of materials and energy between a plant and the outside world. Therefore, the morphology, structure and function of leaves are closely related to the surrounding environment and are most sensitive to the habitat. Accordingly, the structural characteristics of leaves can reflect the adaptation capability of the plant to the environment ${ }^{19}$. Salt secretion is one of the most important mechanisms of plant's salt-tolerance capacity. The salt-secreting structures of plants include glandular hairs, salt glands, vesicles and the waxy coat of the leaf surfaces ${ }^{15}$. However, G. uralensis is dependent on an unique structure, the stomata, to secrete salts out of its body. This study cannot quantify the salt-secreting degree of stomata due to the existence of other salt-secreting structures such as salt glands (Figure $1 d$ ). There are many reports on the salt-tolerance mechanisms of G. uralensis, which include, increasing the content of osmotic adjustment substances, improving the activity of antioxidant enzymes, and accelerating the development of casparian strips on the endodermis of radicles ${ }^{20}$. However, the adaptation strategy to high-salt soils induced by secretion of salts from stomata has not yet been found.

At present, it is not unusual for the use of energy dispersive spectrometry (EDS) to analyse the elements of secretions around stomata. For example, an energy spectrum analysis by Pritchard et al. ${ }^{21}$ shows that the salt crystals 


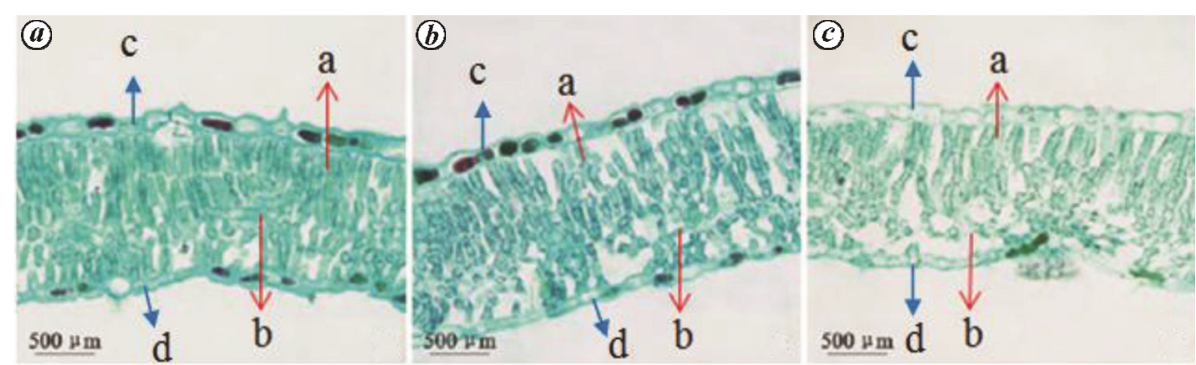

Figure 3. Anatomical characteristics of G. uralensis leaves at different positions. $\boldsymbol{a}$, Upper leaf; $\boldsymbol{b}$, middle leaf; $c$, lower leaf; a, palisade tissue; b, spongy mesophyll; c, upper epidermis; d, lower epidermis.
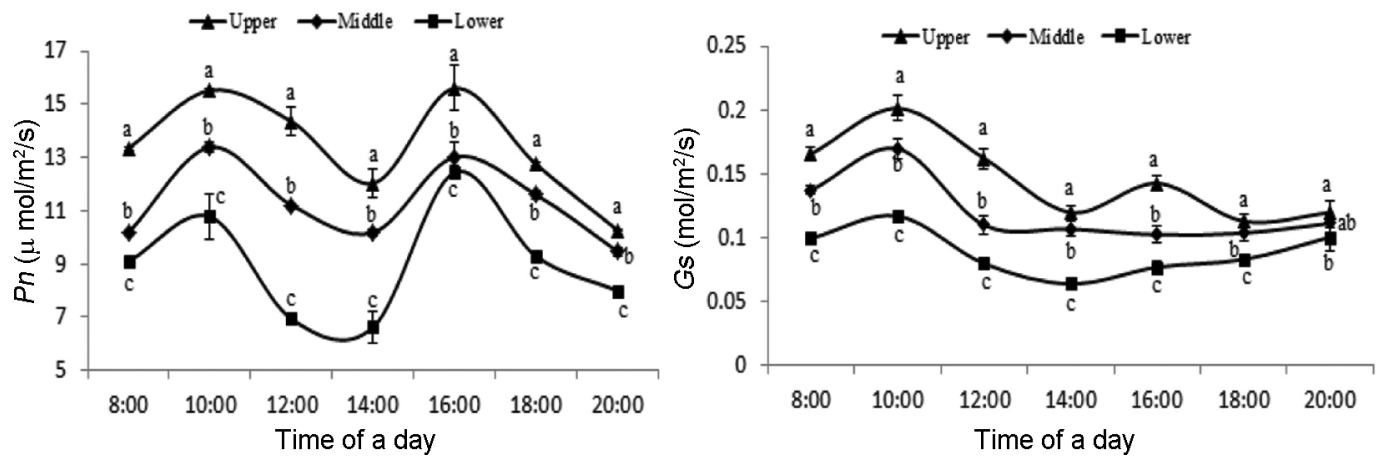

Figure 4. Comparison of net photosynthetic rate and stomatal conductance of G. uralensis leaves at different positions.

around the stomata of long-leaf pine (Pinus palustris Mill.) are mainly calcium sulphate $\left(\mathrm{CaSO}_{4}\right)$. Our EDS indicated a composition of calcium, sulphur, potassium and oxygen (Figure 2). Here sulphate ion seems to be the major balancing anion in the secretions. Potassium is known to be associated with stomatal movement ${ }^{22}$, hence, its presence is not surprising. Calcium is required for the growth of all plants, and is closely related to plant salt tolerance $^{23}$. We speculated that secretions from the stomata of $G$. uralensis would be mainly sulphate, calcium and potassium salts.

Salt stress can lead to physiological drought of plants and affect their morphological structure and physiological functions. In this study, we found that the mesophyll cells in the upper leaves were closely arranged, with welldeveloped palisade tissues. Additionally, the upper leaves also contained largest number of chlorophylls, which could explain why their photosynthetic capacity was the highest. Mesophyll cells in the lower leaves were arranged loosely, and intercellular spaces were large, consistent with the results of a study by Xie et $a .^{24}$.

Photosynthetic pigments are the material basis for leaf photosynthesis, and the content of chlorophyll reflects, to some extent, the growth status of plants and the photosynthetic capacity of the leaves ${ }^{25}$. Salt stress enhances the activity of chlorophyllase, resulting in degradation of chlorophyll $b$ and leading to decreased chlorophyll content; these changes also reduce the photosynthetic capacity of the leaves ${ }^{26}$. In this study, we found that the chlorophyll content in the lower leaves of G. uralensis was significantly lower than that in the upper or middle leaves, which may be due to damage to chloroplasts in the lower leaves, which are subjected to severe salt stress because of the large amounts of salt secreted from the stomata on the lower leaves.

As important structures for $\mathrm{CO}_{2}$ absorption, stomata control the photosynthesis of plants; therefore, variations in stomatal conductance are essential for photosynthe$\operatorname{sis}^{27}$. To avoid the influence of these effects on licorice growth due to obstruction of photosynthetic gas exchange caused by salt-blocked stomata, functional differentiation of stomata occurs. In the present study, the salt-secreting capacity of the stomata on the leaves of different positions was distinct; the stomata on the lower leaves secreted high levels of salts, resulting in the accumulation of larger quantities of salts leading to stomatal blockage. Previous studies have confirmed that the leaf photosynthesis rate is closely related to stomatal conductance ${ }^{28}$; thus, the maximum amount of salt secretion by stomata in the lower leaves resulted in lower stomatal conductance and net photosynthetic rate compared with those of the middle and upper leaves. In contrast, the stomatal conductance and net photosynthetic rate of the upper leaves were highest because stomata did not participate in salt secretion and remained unobstructed. This indicated a balance between salt secretion and photosynthetic gas exchange in the stomata of G. uralensis leaves living in a salt-stressed environment, and that the stomatal function of the leaves at different positions on the plant was distinct. Specifically, stomata of the lower leaves functioned primarily in salt secretion and exhibited lowest stomatal conductance, whereas the stomatal conductance of upper 
leaves was the highest, ensuring regular photosynthetic gas exchange in the licorice plant and the upper leaves did not participate in salt secretion.

In summary, the lower leaves of G. uralensis growing in a salt-stressed habitat were relatively older than the upper and middle leaves, and their capacity for photosynthesis was weak. However, salt secretion capacity of the stomata on the lower leaves was the highest, making these leaves responsible for excreting excess salt and maintaining the internal ion balance. In contrast, stomata of the young leaves found in the middle and upper positions appeared to be involved primarily in photosynthetic gas exchange. The major functions of stomata of licorice leaves at different positions on the plant body were significantly distinct, demonstrating a balance or tradeoff between the two conflicting interests of stomata as an adaptive strategy by $G$. uralensis living in a salt-stressed habitat. Although we found that $G$. uralensis could adapt to the surrounding high-salt environment through stomatal salt secretion, further studies are needed to explore the mechanisms involved in this process.

1. Zhang, W., Lan, F. and Hong, X. X., Chinese Pharmacopoeia, China Medical Science and Technology Press, Beijing, 2015 .

2. Gafner, S. et al., Isoflavonoids and coumarins from Glycyrrhiza uralensis: antibacterial activity against oral pathogens and conversion of isoflavans into isoflavan-quinones during purification. J. Nat. Prod., 2011, 74, 2514-2519; doi:10.1021/np2004775.

3. Saha, S. et al., Structural features and in vivo antitussive activity of the water extracted polymer from Glycyrrhiza glabra. Int. J. Biol. Macromol., 2011, 48, 634-638; doi:10.1016/j.ijbiomac. 2011.02.003.

4. Yazdi, A., Sardari, S. and Sayyah, M., Evaluation of the anticonvulsant activity of the leaves of Glycyrrhiza glabra var. glandulifera grown in Iran, as a possible renewable source for anticonvulsant compounds. IJPR, 2011, 10, 75-82.

5. Wang, J., Chen, X. Q. and Wang, W., Glycyrrhizic acid as the antiviral component of Glycyrrhiza uralensis Fisch. against coxsackievirus A16 and enterovirus 71 of hand foot and mouth disease. $J$. Ethnopharmacol., 2013, 147, 114-121; doi:10.1016/j.jep.2013. 02.017 .

6. Kwon, H. J. et al., In vitro anti-rotavirus activity of polyphenol compounds isolated from the roots of uralensis. Bioorgan. Med. Chem., 2010, 18, 7668-7674.

7. Shetty, T. K., Satav, J. G. and Nair, C. K. K., Protection of DNA and microsomal membranes in vitro by Glycyrrhiza glabra L. against gamma irradiation. Phytother. Res., 2002, 16, 576-578; doi:10.1002/ptr.927.

8. Cai, H., Chen, X. and Zhang, J. B., $18 \beta$-glycyrrhetinic acid inhibits migration and invasion of human gastric cancer cells via the ROS/PKC- $\alpha /$ ERK pathway. J. Nat. Med., 2017, 72, 252-259; doi:10.1007/s11418-017-1145-y.

9. Zhao, K. F. and Feng, L. T., Chinese Halophyte Resources, Science Press, Beijing, 2001.

10. Bhatt, A. and Santo, A., Effects of photoperiod, thermoperiod and salt stress on Gymnocarpos decandrus seeds: potential implications in restoration ecology activities. Botany, 2017, 95, 1093 1098.
11. Pan, Y., Wu, L. J. and Yu, Z. L., Effect of salt and drought stress on antioxidant enzymes activities and SOD isoenzymes of liquorice (Glycyrrhiza uralensis Fisch). Plant Growth Regul., 2006, 49, 157-165.

12. Belin, C., Thomine, S. and Schroeder, J., Water balance and the regulation of stomatal movements. In Abiotic Stress Adaptation in Plants (eds Pareek, A., Sopory, S. and Bohnert, H.), Springer, The Netherlands, 2009.

13. Melotto, M. et al., Plant stomata function in innate immunity against bacterial invasion. Cell, 2006, 126, 969-980; doi:0.1016/ j.cell.2006.06.054.

14. Liu, M. H. et al., Influence of leaf size of plant on leaf transpiration and temperature in arid regions. Chin. J. Plant Ecol., 2013, 37, 436-442; doi:10.3724/SP.J.1258.2013.00045.

15. Wei, C. X., Wang, J. B. and Chen, Y. F., Epicuticular wax of leaf epidermis: a functional structure for salt excretion in a halophyte Puccinellia tenuiflora. Acta Ecol. Sin., 2004, 24, 2451-2456.

16. Khan, N. A., NaCl-inhibited chlorophyll synthesis and associated changes in ethylene evolution and antioxidative enzyme activities in wheat. Biol. Plantarum., 2003, 47, 437-440; doi:10.1023/ b:biop.0000023890.01126.43.

17. Bao, S. D., Soil Agricultural Chemistry Analysis, China Agricultural Press, Beijing, 2000.

18. Callejas, R. et al., Evaluation of a non-destructive method to estimate the concentration of chlorophyll in leaves of table grape cv. Idesia (Arica), 2013, 31, 19-26.

19. He, N. P. et al., Variation in leaf anatomical traits from tropical to cold-temperate forests and linkage to ecosystem functions. Funct. Ecol., 2017, 32, 10-19; doi:10.1111/1365-2435.12934.

20. Koster, P. et al., The battle of two ions: $\mathrm{Ca}^{2+}$ signalling against $\mathrm{Na}^{+}$stress. Plant Biol., 2019, 21, 39-48; doi:10.1111/plb.12704.

21. Pritchard, S. G. et al., Calcium sulfate deposits associated with needle substomatal cavities of container-grown longleaf pine (Pinus palustris) seedlings. Int. J. Plant Sci., 2000, 161, 917-923.

22. Michael, F. R. et al., How can stomata contribute to salt tolerance? Ann. Bot-London, 1997, 80, 387-393.

23. Hao, J. B., Zhang, F. S. and Tian, C. Y., Halophytes in Xinjiang, Science Press, Beijing, 2006.

24. Xie, Z. C., Luo, D. and Zhang, W. J., Effects of silicon on cell microscopic structure under salt stress of Glycyrrhiza uralensis. $J$. Chin. Med. Mater., 2016, 39, 2698-2701; doi:10.13863/ j.issn1001-4454.2016.12.006.

25. Jothiramshekar, S. et al., Responses of selected $\mathrm{C} 3$ and $\mathrm{C} 4$ halophytes to elevated $\mathrm{CO}_{2}$ concentration under salinity. Curr. Sci., 2018, 115, 129-135.

26. Mehta, P., Jajoo, A. and Mathur, S., Chlorophyll a fluorescence study revealing effects of high salt stress on Photosystem II in wheat leaves. Plant Physiol. Bioch., 2010, 48, 16-20.

27. Joshi, S. C. and Palni, L. M. S., Is dew useful for Himalayan plants? Curr. Sci., 2010, 99, 1434-1439.

28. Wong, S. C., Cowan, I. R. and Farquhar, G. D., Stomatal conductance correlates with photosynthetic capacity. Nature, 1979, 282, $424-426$.

ACKNOWLEDGEMENTS. This study was supported by the National Natural Science Foundation of China (31360047). We thank Editage (www.editage.cn) for their support.

Received 15 September 2018; revised accepted 7 January 2019

doi: $10.18520 / \mathrm{cs} / \mathrm{v} 116 / \mathrm{i} 7 / 1212-1217$ 